\title{
Optimization of a test setup for examining blood damage caused by high shear forces
}

\author{
R. Kortlepel, M.Sc. ${ }^{1}$, Dipl.-Ing. B. Krolitzki ${ }^{2}$, M. Müller, M.Sc. ${ }^{2}$, Prof. Dr.-Ing. B. Glasmacher ${ }^{2}$ \\ ${ }^{1}$ Institute for Multiphase Processes, Leibniz University Hannover, Hannover, Germany, kortlepel@imp.uni-hannover.de \\ ${ }^{2}$ Institute for Multiphase Processes, Leibniz University Hannover, Hannover, Germany
}

\begin{abstract}
If blood pumps are applied in the human body or extracorporeal, blood damage can be caused by shear forces that act on the blood during the delivery. For an accelerated development of blood pumps with help of simulations there is a need for reliable limit values for shear forces. To determine these limit values a shear force test setup was built at the Institute for Multiphase Processes. In the context of the presented master thesis the former test setup peripheral devices were evaluated, possible blood damage mechanisms were considered, blood analysis in different experiments to identify the blood damage caused by different elements were made and peripheral devices were replaced. At the same time the shear chamber was constructional reengineered in a project thesis [1]. We were able to show, that in former works the blood damage caused by test setup peripheral devices had heterodyned the results. Finally the test setup was rebuilt with the results and a test was made. Two findings of the thesis are a reduction of about $60 \%$ of the number of components in the blood-conveying tube system and an application of a new fluid seal.
\end{abstract}

\section{Introduction}

Cause of death of nearly $40 \%$ of the human population of the Western civilisation is cardiovascular diseases. A noninsignificant part of these diseases is cardiac insufficiency [2]. Often the application of an artificial blood pump as a "bridge to transplant" or a temporary heart replacement is indispensable. Also in modern blood pumps blood is exposed to unphysiological shear forces during the delivery and will be damaged in different ways. Possibilities of blood damage are the destruction of erythrocytes, thrombogenesis and the activation of the von Willebrand factor. At the present time a large number of prototypes are required for the development of blood pumps, this is timeconsuming and expensive. To save costs in the future during the development of blood pumps, there could be determined load profiles for blood on virtual prototypes with 3D CAD applications with computer-aided fluid simulations. For a significant forecast of the blood damage caused by shear forces limit values must be determined for the several blood elements.

To determine the limit values a shear chamber test setup was built at the Institute for Multiphase Processes. With the test setup high defined shearing loads from short length, as they appear on blood pumps, could be analysed. The shear chamber is a so-called Searle system. It consists of two coaxial cylinders, between them is arranged a $90 \mu \mathrm{m}$ gap. Through this gap this led the blood, which experience shear forces by rotation of the inner cylinder. Independent from each other a maximum load height of $880 \mathrm{~Pa}$ and a load length up to $1400 \mathrm{~ms}$ could be regulated. By the application of a fluid seal the blood is held in the experiment space and kept away from undefined thermal and flow-induced damage in the region of the bearings. The test setup peripheral devices consist of tubes and tube connectors, a blood perfusion system and a fluid seal system. Experiments with the shear chamber are made in the single pass mode. In Image 1 we can see a model of the shear chamber with shear gap and blood respectively fluid seal branch and drain.

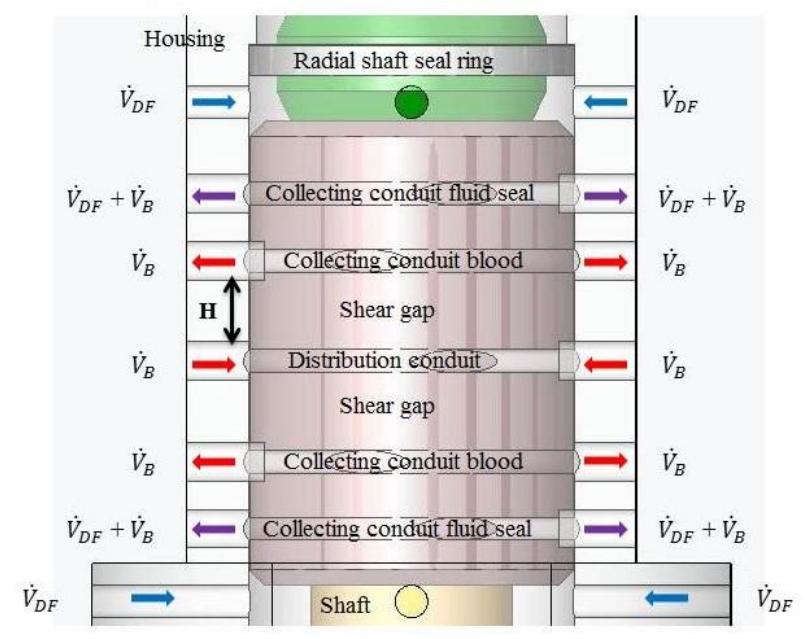

Image 1 Model of the shear chamber $\left(\dot{V}_{D F}\right.$ : Fluid seal flow, $\dot{V}_{B}$ : Blood flow, H: Distance between distribution conduit and collecting conduits). Modified according to [3].

With similar test setups experiments were done on erythrocytes and thrombocytes $[4,5]$.

Aim of the presented master thesis was the optimization of the peripheral devices of the shear chamber test setup with regard to minimizing the blood damage caused by peripheral devices in order to allow a measurement of limit values free from superpositions.

\section{Methods}

In the last configuration of the test setup before this thesis the main weaknesses of the tubes and tube connectors are up to the tube material and the large number of 64 tube connectors. To the tube connectors belong three way 
cocks, Y-pieces and hose connectors, which connect the tube system with the shear chamber. Into the tubes are changes in cross section that results in unphysiological changes in flow.

In seven in each case specific adapted dynamic test setups the blood damage caused by tube material and tube connectors were tested. Depending on the test setup the experiments were made in single pass mode or in one hour mode. The evaluation was made on the basis of the parameters hematocrit, number of thrombocytes and index of hemolysis. For the experiments heparinized porcine blood from the slaughterhouse was used.

In former works a fluid seal based on fluorocarbon was used. This provides the advantage that it separates from blood because of the density difference, if it ends up during the shear chamber experiments in the blood drain. Disadvantage of this fluid seal are high costs. A good alternative is isotonic saline solution. Because it is not possible to separate blood and saline solution again and thinned blood assays deliver wrong results, a clear phase separation between blood and fluid seal in the shear chamber is necessary. Only in this way is it possible to gain a pure blood assay. The possible application of isotonic saline solution as fluid seal was proved in different works, but hitherto the realization was not successful. For the delivery of the saline solution a pump is required, that generates a continual, non-pulsatile volume flow. That allows a clear phase separation. Furthermore the fluid must not come in contact with corrodible parts from the fluid seal system. Finally a test was made with heparinized porcine blood to check the functionality of the optimized shear chamber test setup.

\section{Results}

As the new tube material is used a TygonS50HL tube with $2,4 \mathrm{~mm}$ inner diameter. The tested tube materials exhibit an unremarkable index of hemolysis below $2 \%$. HAYCOX defined that as acceptable for a test duration from one to two hours [6]. But at the one hour experiment the TygonS50HL exhibits a $20 \%$ lower thrombocytes loss (Image 2).

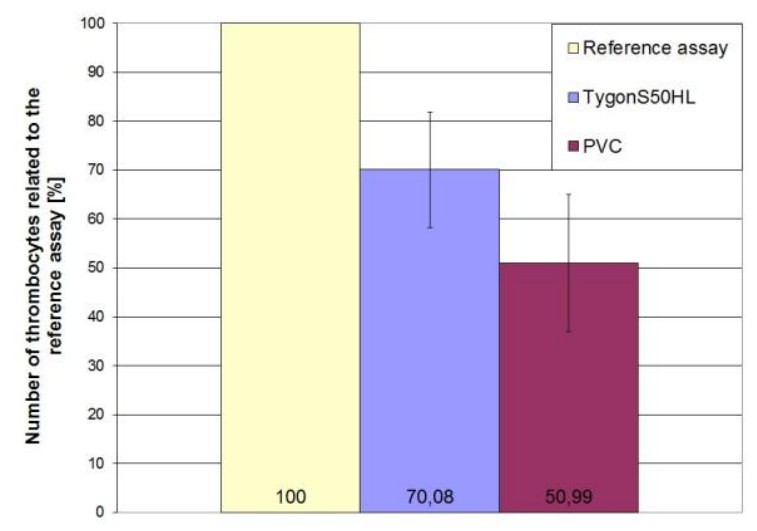

Image 2 Comparison of tube materials: relative number of thrombocytes after one hour blood contact $(n=18)$.
The individual tube connectors have no measurable influence on index of hemolysis and index of thrombocytes. Exemplary Image 3 and 4 show experimental results for hose connectors.

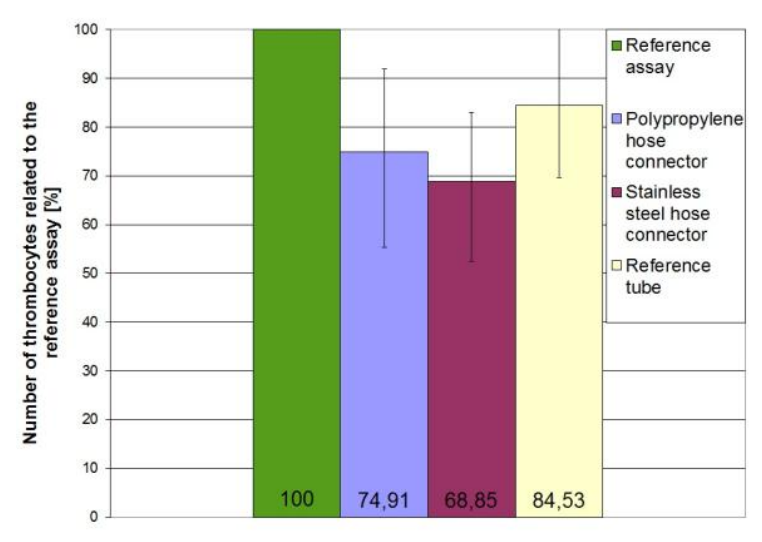

Image 3 Influence of hose connectors on relative number of thrombocytes after one hour blood contact $(n=9)$.

Due to simple acquisition and changeless properties the former used stainless steel hose connectors are replaced by polypropylene hose connectors. Three way cocks can be further used. In several experiments for Y-pieces the problem arose that the blood has after the distribution no equally distributed blood flow. By a modified blood delivery at the optimized test setup the application of Y-pieces can be avoided.

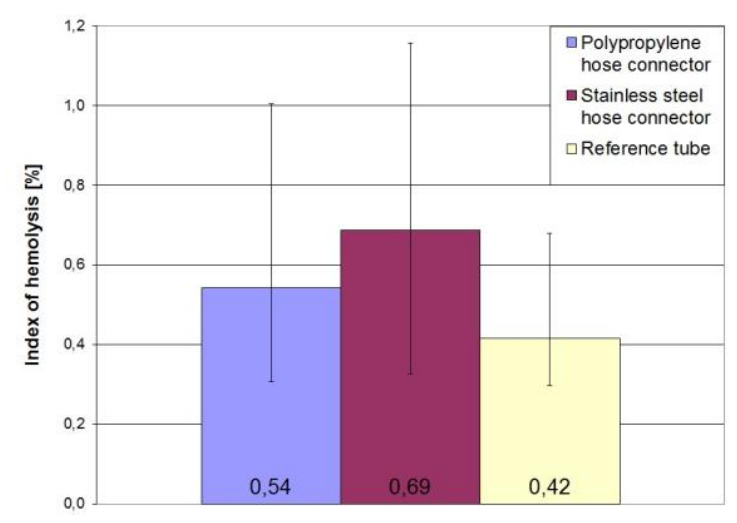

Image 4 Influence of hose connectors on index of hemolysis after one hour blood contact $(n=9)$.

At the new developed fluid seal system the fluid seal is delivered pulsation free with pressurized air. The main part of the system is a pressure chamber with connectors for the fluid seal conduit, the pressurized air conduit and a pressure control valve.

With the gained conclusions the new shear chamber test setup was built. The new test setup consists of three parts: the shear chamber with actuation and bearing, the fluid seal part and the blood-conveying part. Actuation and bearing was adopted from the contemporaneous project 
thesis [1]. The used shear chamber was fabricated at the Institute for Multiphase Processes. The fluid seal delivery system is connected by a tube system with the shear chamber. The assembling of the blood-conveying part is shown in Image 5.

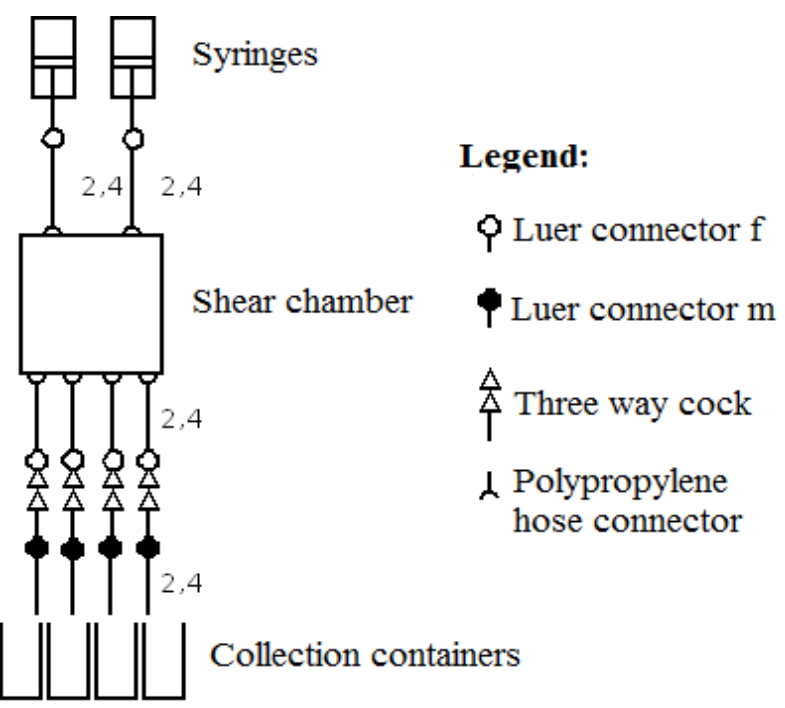

Image 5 Model of the new blood-conveying part with shear chamber, blood perfusion system and tube system.

The two blood branches of the shear chamber are filled with a syringe pump. It is possible to put two syringes at the same time in the syringe pump, so Y-pieces are no longer required.

With the new test setup a test was made with heparinized porcine blood. For the first time a clear phase separation could be regulated during the test by using the new fluid seal delivery system with saline solution. This was shown by measuring the haematocrit before and after the test. The clear phase separation between blood and fluid seal is visible in Image 6 .

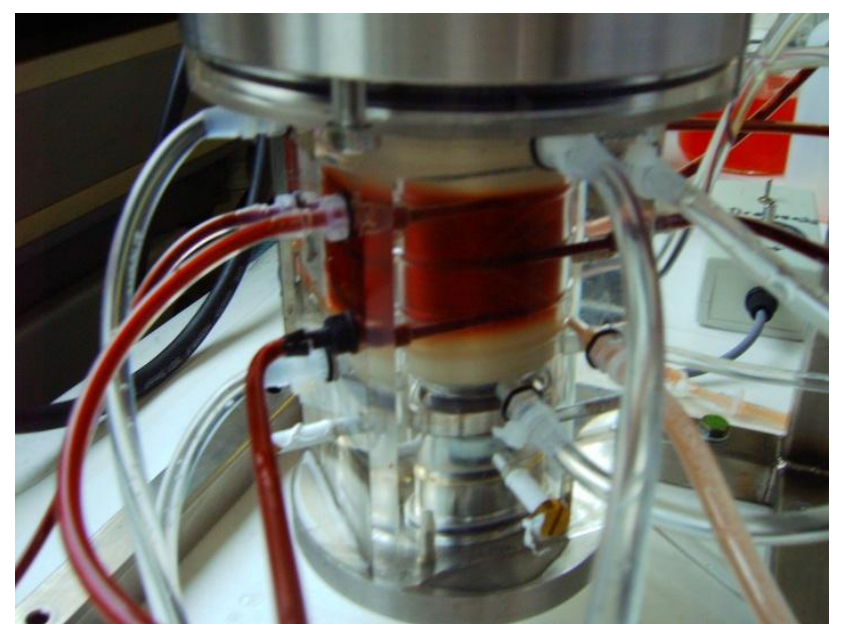

Image 6 Clear phase separation between blood and fluid seal in the shear chamber enabled by the new fluid seal delivery system.
Another result of the thesis is a comparison that was made on the basis of number of components, flow rates, Reynolds numbers and wall shear stresses for the new and the old blood-conveying tube system. The number of tube connectors was reduced by about $60 \%$. By examination of the Reynolds numbers and the wall shear stresses it could be shown that the flow at the new blood-conveying tube system is laminar at flow rates until $140 \mathrm{ml} / \mathrm{min}$ and the expected wall shear stresses could be reduced from 41,3 $\mathrm{Pa}$ on maximum 10,5 $\mathrm{Pa}$.

\section{Conclusion}

All components from the new test setup are tested on their blood damaging effect and exhibit low blood damage. The experiment results were completed by flow viewing. Furthermore a bigger part of blood damage caused by unphysiological flow changes by changes in cross section at test setup could be prohibited by the large reduction of the number of tube connections by about $60 \%$. There is now a totally defined test setup available.

In the literature several times isotonic saline solution was contemplated as fluid seal, by the new designed fluid seal delivery the application is successful for the first time $[3,4,5]$. A continual fluid seal pressure is generated by the delivery with pressurized air, this is of special importance for the clear phase separation. Hereby undiluted blood assays from the shear gap could be gained and the blood could be held securely in his experiment space in the shear chamber. In this way the blood can be kept away from thermal damage caused by the bearings and uncontrollable flow at bearing chamber.

In future works it is possible to start to determine limit values for the different blood elements, like erythrocytes and leucocytes, and the activation of the von Willebrand factor.

\section{Acknowledgements}

This work is partially funded by the German Research Foundation (SFB-TR37 - Q2 and B4) and the cluster of excellence REBIRTH (EXC 62/1).

\section{References}

[1] Wellegehausen, S.-T.: Konstruktive Überarbeitung eines Searle-Systems zur definierten Scherkraftbelastung von Blut, Leibniz University Hannover, unpublished thesis at IMP, 2011

[2] Antretter, H.; Hangler, H.; Höfer, D.; Margreiter, J.; Margreiter, R.; Laufer, G.: Totaler und partieller Herzersatz - Trends und Entwicklungen, Austrian Journal of Cardiology 9 (1-2) (2002), 3-13

[3] Rejnowski, R.: Aufbau und Inbetriebnahme eines Modellsystems zur Untersuchung definierter Bluttraumatisierung, Leibniz University Hannover, unpublished thesis at IMP, 2007 
[4] Paul, R. K.: Untersuchungen zur Blutschädigung durch laminare Strömung im Couette-System, RWTH Aachen, dissertation, 2000

[5] Klaus, S.: Bluttraumatisierung bei der Passage zeitkonstanter und zeitvarianter Scherfelder, RWTH Aachen, dissertation, 2004

[6] Haycox, C. L.; Ratner, B. D.: In vitro platelet interactions in whole human blood exposed to biomaterial surfaces: Insights on blood compatibility, Journal of Biomedical Materials Research 27 (1993), 1181-1193 